\title{
Uso de la tierra y su influencia en la compactación del suelo en el Oriente de Colombia
}

\section{Land use and its influence on soil compaction in eastern Colombia}

\section{Uso da terra e sua influência na compactação do solo no leste da Colômbia}

López Hernández Alejandra ${ }^{1}$, Astroz Cano Andrea Maritza ${ }^{1}$ y Silva Parra Amanda ${ }^{2}$

${ }^{1}$ Ingenieras Agrónomas Universidad de los Llanos y

2Ingeniera Agrónoma, Msc. PhD, Docente Universidad de los Llanos

asilvap@unillanos.edu.co

Recibido 19 de noviembre 2019, Aceptado 12 de marzo 2020

\section{RESUMEN}

En este trabajo, se evalúa el impacto de diferentes usos en la compactación del suelo, para ello se estudiaron cuatro usos considerados como corrientes en la zona de Puerto Lleras-Meta-Colombia: sistema silvopastoril (SSP), monocultivo (MON), pastura (PS) y bosque secundario (BS), se tomó una muestra compuesta de 5 puntos a $20 \mathrm{~cm}$ de profundidad por cada sistema, usándose un diseño experimental irrestrictamente al azar DIA, debido a que los análisis físico-químicos de suelo se analizaron bajo condiciones controladas de laboratorio. Las variables que se evaluaron fueron: Materia orgánica (Walkley and Black), pH (potenciométrico), Densidad aparente (terrón parafinado), Humedad gravimétrica, Humedad volumétrica, Densidad real, Porosidad, Lámina de agua, Humedad volumétrica a capacidad de campo, Macroporosidad, Resistencia a la penetración (penetrologger) y Textura 8bouyoucos). Para evaluar la resistencia a la penetración de cada sistema se utilizó el penetrologger. El análisis estadístico se realizó mediante análisis de variancia $(P<0.01)$ y las diferencias entre los tratamientos se analizaron mediante prueba de Tukey $(P<0.05)$. Los datos se corrieron en paquete estadístico Infostat. Los resultados indicaron que el BS, SSP, PS y MON no presentaron problemas de compactación, medidos por la densidad aparente y la resistencia a la penetración, los sistemas poseen suelos de predominancia arenosos y por lo tanto son menos susceptibles a este problema, el sistema de MON aunque no presentó problema de 
compactación tiende a una macroporosidad (M) cercana al 10\% (14.08\%). EI SSP presentó una Pt total ideal, y el BS es un sistema altamente retenedor de agua.

Palabras clave: Bosque, monocultivo, pastos, silvopastoril, suelo.

\section{ABSTRACT}

In this work, the impact of different uses on soil compaction is evaluated, for which the current and current means uses in the area of Puerto Lleras-Meta-Colombia are studied: silvopastoral system (SPP), monoculture (MON), pasture (PS) and secondary forest (SF), a composite sample of 5 points at $20 \mathrm{~cm}$ of depth was redirected for each system, we used an experimental design absolutely randomly DIA, because the physical-chemical analyzes of the soil were analyzed under controlled laboratory conditions. The variables that were evaluated were: Organic matter (Walkley and Black), pH (potentiometric), Apparent density, Gravimetric humidity, Volumetric humidity, Real density, Porosity, Water sheet, Volumetric humidity at field capacity, Macroporosity, Resistance to Penetration and texture. To assess the resistance to penetration of each of the systems, the penetrator has also been used. The statistical analysis was carried out by means of the analysis of variance $(P<0.01)$ and the differences between the treatments were analyzed by the Tukey test $(P<0.05)$. The data were run in the Infostat statistical package. The results indicate that the SF, SPS, PS and MON do not present problems of compaction, means by the apparent presence and the resistance to the penetration, the systems of predominance of the sandy ones and therefore are less susceptible to this problem, the system However, there was not a problem of compaction that tends to a macroporosity (M) close to $10 \%(14.08 \%)$. The SPS presented a total ideal, and the FS is a highly water retaining system.

Keywords: Forest, monoculture, pastures, silvopastoral, soil.

\section{RESUMO}

Neste trabalho, avalia-se o impacto de diferentes usos na compactação do solo, estudando-se quatro usos considerados correntes na área de Puerto Lleras-Meta- 
Colômbia: sistema silvipastoril (SSP), monocultura (MON), pastagem ( PS) e floresta secundária (BS), uma amostra composta de 5 pontos a $20 \mathrm{~cm}$ de profundidade foi coletada para cada sistema, utilizando um delineamento experimental DIA irrestritamente aleatório, pois as análises físico-químicas do solo foram analisadas sob condições controladas laboratório. As variáveis avaliadas foram: matéria orgânica (Walkley e Black), pH (potenciométrico), densidade aparente (nódulo parafinizado), umidade gravimétrica, umidade volumétrica, densidade real, porosidade, lençol d'água, umidade volumétrica à capacidade de campo, macroporosidade, Resistência à penetração (penetrologger) e Texture (bouyoucos). O penetrologista foi utilizado para avaliar a resistência à penetração de cada sistema. $A$ análise estatística foi realizada por análise de variância $(P<0,01)$ e as diferenças entre os tratamentos foram analisadas pelo teste de Tukey $(P<0,05)$. Os dados foram executados no pacote estatístico Infostat. Os resultados indicaram que as BS, SSP, PS e MON não apresentaram problemas de compactação, medidos pela densidade aparente e resistência à penetração, os sistemas possuem solos predominantemente arenosos e, portanto, são menos suscetíveis a esse problema. Apesar de não apresentar um problema de compactação, tende a uma macroporosidade (M) próxima a 10\% (14,08\%). O SSP apresentava uma Pt total ideal e o BS é um sistema altamente retentor de água.

\section{Palavras-chave:}

\section{INTRODUCCIÓN}

Los sistemas de producción agropecuarios en Colombia y específicamente los de la región de la Orinoquia ubicada en el Oriente de Colombia, se basan principalmente en la explotación de los recursos naturales, a través de la implementación de monocultivos y de ganadería intensiva, ocasionando sobreuso del suelo, degradación y abandono de la tierra (Noruega y Vélez, 2011).

La compactación del suelo en áreas planas de agricultura intensiva en la Orinoquia y la erosión del suelo de ladera, son los principales problemas de degradación en Colombia (Jaramillo, 2002). Los procesos de perdida de estructura, compactación, 
encostramiento superficial están íntimamente asociados con el uso indiscriminado y excesivo de implementos de labranza (Streck et al., 2004). Los sistemas de uso de suelo como los de monocultivo tienden a la degradación y a la insostenibilidad en el largo plazo (Lal, 2015), por lo tanto, hay que hacer un uso más racional de este recurso, para evitar su degradación, implementando sistemas que ayuden a mantener los ecosistemas, como los agroforestales, entre ellos los silvopastoriles.

Según la FAO, (2016) la compactación de suelo puede disminuir el rendimiento agrícola hasta un $60 \%$, lo cual se debe a la mala gestión agrícola $(80 \%)$ y el sobrepastoreo (16\%), son las dos principales causas antrópicas de compactación, ocasionando la disminución de la macroporosidad del suelo y aumento de su microporosidad, ofreciendo una mayor resistencia a la penetración de las raíces de las plantas. La labranza mínima está entre las mejores opciones para reducir gastos de laboreo, conservar suelos y agua, incrementar la disponibilidad de carbono orgánico del suelo y reducir las emisiones netas de $\mathrm{CO}_{2}$ y otros gases de efecto invernadero (GEI). Se estima que el $4 \%$ de la superficie terrestre global se encuentra compactada (Alakukku et al., 2003).

Los suelos se diferencian en propiedades químicas como su textura, contenido de agua, materia orgánica, y propiedades físicas como la densidad aparente, porosidad, y resistencia a la penetración, las cuales se pueden ver afectados por la compactación, es así que Blainski et al. (2008) afirmaron que la pérdida de suelo por erosión, reducción de la materia orgánica y la compactación son algunos de los factores que corroboran la degradación física del suelo.

Según Luciano et al., (2012) el uso de maquinaria en suelos con una humedad próxima al límite de plasticidad es uno de los principales factores que compacta los suelos agrícolas, pues el agua reduce la cohesión de las partículas del suelo. La implementación de pasturas mejoradas con manejo intensivo, principalmente del género Brachiaria, es otro uso común en esta zona, el correcto manejo de pasturas es primordial para garantizar un sistema de producción sustentable. Sin embargo, el manejo de maquinaria, y el sobrepastoreo puede ocasionar problema de compactación (Mur y Balbuena, 2014). 
El suelo como recurso natural es primordial para la sostenibilidad de los sistemas productivos de la región, por lo que éste se convierte en elemento principal en el desarrollo de sistemas agroecológicos, agrícolas y pecuarios (Medina, 2016). Los principales problemas de la degradación física de los suelos son la compactación y el adensamiento, estos causan fuertes disminuciones en los rendimientos de los cultivos (Lal, 2015).

La compactación del suelo, debido al colapso o disminución de los espacios de poros, es la causa más común de restricción física para el crecimiento y desarrollo de las raíces, la cual puede ser identificada mediante la medición de la densidad aparente y la resistencia a la penetración (Jaramillo, 2002).

La degradación de la estructura del suelo es considerada como el tipo de degradación de tierras más difícil de localizar y reconvertir; medible mediante la resistencia a la penetración, la razón es que esta degradación es un fenómeno subsuperficial (Medina, 2016). De ahí, que sea necesario identificar los usos del suelo en la zona de estudio, con impactos negativos en las propiedades físicas de los suelos, como indicadores de compactación.

La compactación del suelo se define como la pérdida de volumen que experimenta una determinada masa de suelo, debido a fuerzas externas que actúan sobre él (McKenzie y Ramos, 2010). En condiciones naturales (sin intervención antrópica) se pueden encontrar horizontes con diferentes grados de compactación, lo que se explica por las condiciones que dominaron durante la formación y la evolución del suelo. Sin embargo, es bajo condiciones de uso agrícola intensivo o sistemas de monocultivo, que este fenómeno se acelera y llega a producir serios problemas en el desarrollo de las plantas cultivadas. El suelo ideal es aquel que se compone de un $50 \%$ de sólidos, $25 \%$ de agua y $25 \%$ de aire, características que pueden encontrarse en sistemas naturales como bosques (McKenzie y Ramos, 2010)

Según Lima et al., (2004) "los sistemas de pasturas, la intensidad del pisoteo y el tiempo de permanencia de los animales en el área también determinan el grado de 
degradación estructural que puede ocurrir al suelo". Los bovinos ejercen una fuerza de hasta $400 \mathrm{kPa}$ (kilopascales) en la superficie del suelo.

Camargo y Alleoni, (1997) reconocen cuatro formas y medidas de la compactación del suelo: a) densidad del suelo o porosidad; b) infiltración del agua; c) resistencia a la penetración y; d) evaluación en calicatas. La densidad del suelo es el método más indicado por ser menos influenciado por factores externos, como la humedad. Al contrario, la infiltración del agua y la resistencia a la penetración son sensibles al estado de humedad del suelo. El diagnostico de abertura de calicatas es más recomendable para evaluar el perfil del suelo al final de un cultivo.

Condiciones de crecimiento favorables, tales como propiedades físicas adecuadas, adecuada precipitación y una óptima fertilidad, pueden minimizar los efectos de la compactación (Bertol et al., 2000). Una compactación severa puede causar una pérdida de rendimiento de hasta un $60 \%$, sin embargo, se estima que en promedio la compactación reduce el potencial de rendimiento entre un 10 a 20\%.

Una gestión adecuada del suelo constituye un factor esencial en la agricultura sostenible y proporciona también un resorte valioso para regular el clima y salvaguardar los servicios ecosistémicos y la biodiversidad. Los suelos saludables son un requisito previo básico para satisfacer las diversas necesidades de alimentos, biomasa (energía), fibra, forraje y otros productos, y para garantizar la prestación de los servicios ecológicos esenciales en todas las regiones del mundo (FAO, 2015). Las funciones específicas que un suelo proporciona se rigen en gran medida por el conjunto de propiedades químicas, biológicas y físicas que se hallan en dicho suelo. Así mismo, los suelos son una reserva clave de biodiversidad mundial que abarca desde los microorganismos hasta la flora y la fauna. Esta biodiversidad tiene una función fundamental en el respaldo a las funciones del suelo y, por tanto, a los bienes y servicios ecosistémicos asociados a estos (FAO, 2015).

Según el IGAC, (2013) el área continental se encuentra cubierta en su mayoría por bosques, en un 53\% del área total, seguido por los territorios ganaderos que corresponden a un $31 \%$, y los territorios agrícolas a un $5 \%$. 


\section{METODOLOGÍA}

\section{Ubicación}

El trabajo se realizó en el municipio de Puerto Lleras-Meta, localizado al oriente de Colombia, con coordenadas geográficas Latitud: 3.267 , Longitud: $-73.3673^{\circ} 16^{\prime} 1 "$ Norte, $73^{\circ} 22^{\prime} 1^{\prime \prime}$ Oeste. Los veranos son cortos muy caliente y nublado, temperatura promedio $30^{\circ} \mathrm{C}$, velocidad del viento $6 \mathrm{Km} / \mathrm{h}, 80 \%$ de humedad relativa y precipitación anual de $2611 \mathrm{~mm}$. En Puerto Lleras existen cuatro unidades geomorfológicas: el primero es la planicie aluvial activa de desborde $(8,1 \%)$, después están los suelos de terrazas aluviales, los suelos de altillanura $(59,3 \%)$ y las formas subordinadas a la red hidrográfica. De estas, la planicie aluvial es la de mayor fertilidad, presenta paisajes ubicados en clima cálido húmero, cada uno con subpaisajes fisiográficos de acuerdo con su morfología, litología y edad. Se pueden resumir así: 1. Altiplanicie Estructural - Erosional (LDel-2, LCcd1, LBbc, LAab, LEab, APa y VCa). Presenta colinas y lomas arcillolitas, conglomerados, superficies onduladas, altillanura plana, vallecitos aluviales con erosión moderada y ligera, (IGAC, 2015; Mintrabajo, 2013).

\section{Diseño estadístico}

Se estudiaron cuatro diferentes usos del suelo, considerados como comunes en la zona de estudio, los cuales presentaron similares condiciones de formación de los suelos, clasificados como Inceptisoles, pero se distinguieron en cuanto al propósito de la producción, manejo, vegetación y tiempo de explotación.

Se aplicó un diseño irrestrictamente al azar DIA, con cuatro tratamientos y cinco repeticiones para un total de 20 unidades experimentales. La Tabla 1 describe los sistemas de uso seleccionados.

\section{Ensayo de campo}

Se seleccionaron cinco puntos totalmente al azar en cada sistema y se establecieron cajuelas tomando muestras a $20 \mathrm{~cm}$ de profundidad, extrayendo 3 submuestras compuestas, que correspondieron a las repeticiones por cada 
tratamiento en un área de $100 \mathrm{~m}^{2}$, las cuales fueron empacadas y rotuladas debidamente. Para el estudio de la resistencia a la penetración se realizó directamente en cada uno de los sistemas, usando el instrumento penetrologger, en el cual se realizaron cinco mediciones en los puntos, donde se tomaron las muestras del suelo en cada sistema.

Tabla 1. Tratamientos evaluados

\begin{tabular}{|c|c|c|}
\hline Tratamientos & Sistemas & Descripción \\
\hline T1 & $\begin{array}{l}\text { Silvopastoril. } \\
\text { (SSP) }\end{array}$ & $\begin{array}{l}\text { El sistema silvopastoril está compuesto por } \\
\text { pastura de Brachiaria decumbens asociado con } \\
\text { árboles nativos del género leguminosas, su } \\
\text { tiempo de uso es de } 15 \text { años. Sistema extensivo, } \\
\text { y no hay manejo del pastoreo. }\end{array}$ \\
\hline T2 & $\begin{array}{l}\text { Monocultivo. } \\
\text { (MON) }\end{array}$ & $\begin{array}{l}\text { El sistema de monocultivo está plantado con } \\
\text { Manihot esculenta, su tiempo de uso es de } \\
\text { aproximadamente } 1 \text { año. }\end{array}$ \\
\hline T3 & $\begin{array}{l}\text { Pastura. } \\
\text { (PAS) }\end{array}$ & $\begin{array}{l}\text { El sistema de pastura está sembrado con pasto } \\
\text { Brachiaria decumbens, } 5 \text { años de uso. Sistema } \\
\text { extensivo y no hay manejo del pastoreo. }\end{array}$ \\
\hline T4 & $\begin{array}{l}\text { Bosque } \\
\text { secundario } \\
\text { (BS) }\end{array}$ & $\begin{array}{l}\text { El bosque secundario es una combinación de } \\
\text { varias especies de la zona, está en sucesión } \\
\text { natural, y lleva aproximadamente } 12 \text { años. }\end{array}$ \\
\hline
\end{tabular}

\section{Variables físico-química analizadas}

Las muestras de suelo se procesaron en el Laboratorio de Suelos de la Universidad de los Llanos, correspondiendo a análisis físico-químicos, se siguieron las metodologías consignadas por el IGAC 2006. (Tabla 2)

\section{Análisis estadísticos}

El análisis estadístico se realizó mediante análisis de varianza $(P<0.01)$ y las diferencias entre los tratamientos se analizaron mediante prueba de Tukey $(P<0.05)$. Los datos se corrieron usando en paquete estadístico Infostat (González, et al., 2008). 
Tabla 2. Metodología utilizada para la evaluación de las variables físicas en el presente estudio.

\begin{tabular}{ccc}
\hline Variable & Unidad & Metodología \\
\hline Densidad aparente $(\mathrm{Da})$ & $\begin{array}{c}\mathrm{g} / \mathrm{cc} \\
\text { \%arena, limo, } \\
\text { arcilla }\end{array}$ & Terrón Parafinado \\
Textura & & Bouyoucos \\
$\mathrm{pH}$ & $\%$ & Peachimetro \\
Materia orgánica & $\mathrm{MPa}$ & Walkley and Black \\
Resistencia a la penetración & $\mathrm{Mm}$ & $\mathrm{Penetrologger}$ \\
Lámina de agua $(\mathrm{Z})$ & $\%$ & $\mathrm{H} \theta / 100 \times$ prof $(\mathrm{mm})$ \\
Porosidad Total $(\mathrm{Pt})$ & $\%$ & $1-\mathrm{Da} / \mathrm{Dr} \times 100$ \\
Humedad Volumétrica $(\mathrm{H} \theta)$ & $\%$ & $\mathrm{Hw} \times \mathrm{Da}$ \\
Humedad Gravimétrica $(\mathrm{Hw})$ & & Psh-pss $/ \mathrm{pss} \times 100$ \\
\hline
\end{tabular}

\section{RESULTADOS Y DISCUSIÓN}

\section{Efecto del uso del suelo en la densidad, pH y materia orgánica (MO)}

La densidad aparente (Da) hasta los $20 \mathrm{~cm}$ de profundidad varió de 1,15 a 1,32 $\mathrm{gr} / \mathrm{cm}^{3}$ en BS y MON, lo que indica que existe variación en esta propiedad, de acuerdo al sistema que este establecido. Laureda et al., (2016), afirman que la intensificación de la producción es debida a los monocultivos, trayendo como resultado la utilización excesiva de maquinaria, pudiendo ocasionar compactación por "pie de arado" y elevando la Da. BS presentó diferencia con MON y SSP $(P<0.05)$, pero fue igual a PS $(P>0.05)$, presentando los valores más bajos, sin embargo, PS también fue igual a MON y SSP con valores más altos $(P>0.05)$ (Tabla 3). De acuerdo con los rangos establecidos por Jaramillo (2002), en suelos arenosos se presenta compactación, cuando la $\mathrm{Da}>1.6 \mathrm{~g} / \mathrm{cc}$, lo cual demuestra que los usos de suelo evaluados, con texturas predominantemente arenosas presentan compactación. Generalmente, las prácticas de manejo tienen mayor impacto sobre las propiedades físicas de suelos arenosos que en suelos arcillosos (Silva et al., 2011). 
Los suelos arenosos tienden a compactarse menos que los arcillosos, ya que, puesto que la compactación es una reducción de la macroporosidad del suelo, caracterizándose los de textura arenosa por su mayor predominancia de macroporos, por lo tanto, tienen un buen drenaje y menor fuerza de cohesión, conservando así su estructura y perdiendo en menor grado el espacio poroso (Silva et al., 2011).

Tabla 3. Efecto de los sistemas de uso en la densidad aparente del suelo.

\begin{tabular}{cccc}
\hline Sistemas & $\mathbf{D a}\left(\mathbf{g} / \mathbf{c m}^{3}\right)$ & $\mathbf{p H}$ & MO (\%) \\
\hline Monocultivo & $1.32 \pm 0.30^{\mathrm{b}}$ & $5.54 \pm 0.23^{\mathrm{b}}$ & $1.18 \pm 0.11^{\mathrm{b}}$ \\
Silvopastoril & $1.31 \pm 0.30^{\mathrm{b}}$ & $4.68 \pm 0.23^{\mathrm{ab}}$ & $1.10 \pm 0.11^{\mathrm{b}}$ \\
Pastura & $1.23 \pm 0.30^{\mathrm{ab}}$ & $4.44 \pm 0.23^{\mathrm{a}}$ & $1.38 \pm 0.11^{\mathrm{ab}}$ \\
Bosque & $1.15 \pm 0.30^{\mathrm{a}}$ & $4.16 \pm 0.23^{\mathrm{a}}$ & $1.70 \pm 0.11^{\mathrm{a}}$ \\
\hline
\end{tabular}

$0.30=$ Error estándar. Medias con letras diferentes son significantes $(p>0,05)$, prueba de Tukey. $\mathrm{Da}=$ densidad aparente. $\mathrm{MO}=$ materia orgánica.

El pisoteo excesivo por los animales en sistemas de pastoreo; $y$ en el monocultivo, el uso de maquinaria pesada, la mecanización en condiciones inadecuadas de humedad, aumenta la densidad aparente a niveles mayores de $1.0 \mathrm{~g} / \mathrm{cm}^{3}$ (Batey y McKenzie, 2006). Los factores anteriormente relacionados en el SSP $\left(1,31 \mathrm{~g} / \mathrm{cm}^{3}\right)$, como en el sistema de MON $\left(1,32 \mathrm{~g} / \mathrm{cm}^{3}\right)$ pudieron ser influyentes en la obtención de valores más altos de Da del suelo (Tabla 3).

La densidad aparente como indicador de la compactación es mayor a medida que aumenta la carga animal, estos resultados concuerdan también con lo afirmado por Foloni et al., (2003) puesto que un suelo sometido a una determinada presión, ocasiona una reducción en su volumen y como consecuencia trae un aumento en su densidad aparente. En el SSP comparado con la PAS, se manejan ambos de manera extensiva, y no se realiza un manejo del pastoreo adecuado, como la rotación de potreros, tendiendo ambos a problemas de compactación por lo tanto alcanzaron el nivel crítico de densidad aparente de 1.31 y $1,23 \mathrm{~g} / \mathrm{cm}^{3}$, respectivamente (Tabla 3 ). 
La compactación ocasionada por el pisoteo del animal depende principalmente de la clase de suelo, del contenido de humedad, carga animal y de la especie forrajera, siendo generalmente localizada en áreas de mayor movimiento como búsqueda de agua o alimento (Lima et al., 2004).

Los sistemas PAS, BS y MON, clasifican como suelos ácidos, sin diferencia entre ellos ( $P>0.05$ ), aunque MON tiende a menor acidez activa, con un $\mathrm{pH}$ moderado de 5.54 , siendo mayor su valor $(\mathrm{P}<0.05)$ en comparación con PS y BS (Tabla 3$)$. Este resultado se puede deber a que el MON recibe constantemente prácticas de encalado y como es lógico tienen su efecto en el aumento del pH (Fageria y Baligar, 2008), y en los SSP por su parte se tiene comprobado que los árboles leguminosos implementados en las pasturas mejoran el pH del suelo, porque aportan N, bajando los costos por fertilizantes nitrogenados y los impactos ambientales (Lüscher et al., 2014; Parra et al., 2019).

Los valores del porcentaje de la MO variaron de 1,10 a 1,70\%, siendo el SSP el de menos contenido y el sistema de BS el de mayor contenido, se observó un comportamiento similar en el contenido de $\mathrm{MO}$ y $\mathrm{Da}$, es decir, que, entre los SSP, MON y PAS no se encontraron diferencias $(P>0.05)$, sin embargo, PAS y BS, también fueron similares, siendo este el sistema el de mayor contenido de materia orgánica (1,70\%) (Tabla 3). Sin embargo, la influencia del uso de suelo en la MO no es significativa, debido a que los valores obtenidos, clasifican como suelos pobres de materia orgánica menos de $2 \%$, que es común en de suelos de esta zona tropical. Resultados similares en el BS, fueron obtenidos por Cairo et al., (2017), con incrementos del contenido de materia orgánica en un bosque, debido al aporte de la hojarasca.

El alto contenido de materia orgánica en los pastos cultivados podría atribuirse a alta cantidad de residuos, principalmente de gramíneas sobre la superficie del suelo. Según Moussadek et al. (2014), esta retención de residuos en la superficie del suelo aumenta el carbono orgánico y agregados estables en agua. Según Orellana et al., (2008) los residuos de gramíneas presentan altos contenidos de lignina, con una 
estrecha relación entre carbono/nitrógeno permitiendo una lenta mineralización y enriquecimiento de la materia orgánica.

Los cambios en el stock de carbono se presentan de manera diferente, según sea el tipo de uso del suelo; por ejemplo, se espera que, en suelos de bosque, la distribución de carbono en profundidad sea menor en comparación con el que están establecidos matorrales o pasturas. Así, el stock de $C$ en los suelos de bosque es sensible al cambio por diferentes factores, entre los que se encuentra el clima (Palosuo, 2008).

Lo anterior coincide con los resultados de Rodríguez, (2016) quien encontró que el mayor stock de $\mathrm{C}$ a más profundidad en los sistemas agrícolas asociados con pasturas. Por su parte en el SSP se observa que los valores fueron muy bajos, al respecto, Silva et al., 2011, menciona que los contenidos de carbono orgánico (CO) en este sistema tienen que ver con la mezcla de leguminosas rastreras, que incrementaron paulatinamente el $\mathrm{CO}$ con el tiempo de explotación, así que los convierte en alternativas viables, pero en el largo plazo.

\section{Contenidos de arena, limo y arcilla en los diferentes usos del suelo.}

La textura es aquella propiedad que establece las cantidades relativas en que se encuentran las partículas de diámetro menor a $2 \mathrm{~mm}$, es decir, la tierra fina, en el suelo; estas partículas, llamadas separados, se agrupan en tres clases, por tamaños: Arena $(A)$, Limo (L) y Arcilla (Ar).

Se registra que el menor contenido de arena en cada uno de los usos evaluados, corresponde a BS, con $52,0 \%$ de arena, seguido por el MON con un $62,80 \%$, siendo similares y los sistemas con mayor porcentaje de arena en orden creciente fueron SSP, PS, este último presentó $72,40 \%$, por encima de 20 puntos porcentuales comprado con BS (Tabla 4).

Los valores de \% de Arena, limo y arcilla encontrados en los diferentes sistemas de uso según London (2014), clasificarían como Arenosa Franca para PAS, Franco Arenoso para SSP y MON y Franco-limoso para BS (Tabla 4), indicando que son 
suelos de buena infiltración y baja resistencia a la penetración. Sin embargo, no se puede afirmar que en suelos arenosos no existe compactación, puesto que en valores $>1.6 \mathrm{~g} / \mathrm{cm}^{3}$. Los resultados de porcentaje de arena, limo y arcilla para cada uno de los sistemas, puede llegarse a la conclusión que los suelos arenosos, son menos susceptibles a problemas de compactación que suelos arcillosos. Los bajos porcentajes de arcilla, pueden estar relacionados con procesos de iluviación de arcillas, o procesos de erosión característico de Inceptisoles (Jaramillo, 2002), asociados a las altas precipitaciones, donde el impacto de la gota de lluvia sobre el suelo, también puede ocasionar sellamiento y dispersión de arcillas.

Tabla 4. Contenido de arena, limo y arcilla (\%) en los diferentes usos del suelo.

\begin{tabular}{ccccc}
\hline Tratamiento & $\begin{array}{c}\text { Arena } \\
(\mathbf{A})\end{array}$ & $\begin{array}{c}\text { Limo } \\
(\mathbf{L})\end{array}$ & $\begin{array}{c}\text { Arcilla } \\
(\mathbf{A r})\end{array}$ & $\begin{array}{c}\text { Grado de } \\
\text { textura }\end{array}$ \\
\hline Pastura & $72.40 \pm 2.90^{\mathrm{b}}$ & $23.201 .71^{\mathrm{ab}}$ & $4.40 \pm 1.67^{\mathrm{b}}$ & Arenosa-franca \\
Silvopastoril & $70.00 \pm 2.90^{\mathrm{b}}$ & $20.40 \pm 1.71^{\mathrm{a}}$ & $9.60 \pm 1.67 \mathrm{a}^{\mathrm{b}}$ & Franco-arenosa \\
Monocultivo & $62.80 \pm 2.90 \mathrm{a}^{\mathrm{b}}$ & $29.20 \pm 1.71^{\mathrm{b}}$ & $7.60 \pm 1.67 \mathrm{a}^{\mathrm{b}}$ & Franco-arenosa \\
Bosque & $52.00 \pm 2.90^{\mathrm{a}}$ & $36.80 \pm 1.71^{\mathrm{c}}$ & $11.20 \pm 1.67^{\mathrm{a}}$ & Franco-_limosa \\
\hline
\end{tabular}

Error estándar $=A: 2.90, L: 1.71, A r: 1.67$. Medias con una letra diferente tienen una significancia $(p$ $>0,05)$. Prueba de Tukey

Según Hakansson et al., (1988) el sistema de preparación convencional del suelo ocasiona compactación subsuperficial, por la descompactación y movilización mecánica de arcillas, al mismo tiempo en que la carga aplicada presenta efecto acumulativo con los años, ocurriendo una alteración de partículas sin variación de su volumen. Los sistemas de manejo del suelo tienen gran influencia en sus propiedades físicas y están relacionadas con la compactación (Lima et al., 2004; Streck et al., 2004). EI BS presento diferencias significativas con los demás sistemas $(\mathrm{P}<0.05)$, presentando valores ideales de porcentaje de arena limo y arcilla, clasificado como un franco limoso, indicando que de acuerdo con su textura es un sistema de más alta fragilidad.

El sistema radicular permite a las plantas sujetarse al suelo y adquirir agua y nutrientes necesarios para realizar sus funciones vitales, en primera instancia; al 
mismo tiempo, desempeñan una función ecológica importante porque su estructura forma una especie de malla que protege al suelo, evitando que se desprenda ante los elementos que lo golpean; adicionalmente, al adherirse a sus partículas, lo mantienen unido, como si tuviese una especie de pegamento y, por último, absorben el exceso de agua que al acumularse debilita el terreno haciendo que se desprenda, como en el caso de las pendientes inclinadas (Valdés, 2010).

\section{Efecto de humedad gravimétrica, volumétrica, lámina de agua y porosidad}

Los usos BS, MON, SSP y PS no influenciaron $(P>0.05)$, en la humedad gravimétrica, volumétrica y lámina de agua medidas a capacidad de campo, sin embargo, se puede notar que el BS, posee mayor almacenamiento de agua, (tabla 5). Según Tavares Filho y Tessier (2009), la compactación del suelo es más perjudicial en suelo seco, puesto que en condiciones de mayor contenido de agua puede haber crecimiento radicular en valores de resistencia del suelo a la penetración superiores a 4,0 MPa.

Tabla 5. Efecto de la humedad gravimétrica $(\mathrm{Hwcc})$ y volumétrica $(\mathrm{H} \theta \mathrm{cc})$, lámina de agua a capacidad de campo (Zcc), porosidad total (Pt) y macroporosidad (M).

\begin{tabular}{cccccc}
\hline Tratamiento & $\begin{array}{c}\text { Hwcc } \\
(\%)\end{array}$ & $\begin{array}{c}\text { H } \theta \mathbf{c c}, \mathbf{m} \\
(\%)\end{array}$ & Zcc (mm) & $\begin{array}{c}\mathbf{P t} \\
(\%)\end{array}$ & $\begin{array}{c}\mathbf{M} \\
(\mathbf{m m})\end{array}$ \\
\hline Bosque & $29.20 \pm 3.47^{\mathrm{a}}$ & $33.78 \pm 4.16^{\mathrm{a}}$ & $67.42 \pm 8.13^{\mathrm{a}}$ & $56.39 \pm 1.03^{\mathrm{b}}$ & $22.61 \pm 8.13^{\mathrm{a}}$ \\
Monocultivo & $27.17 \pm 3.47^{\mathrm{a}}$ & $37.33 \pm 4.16^{\mathrm{a}}$ & $72.42 \pm 8.13^{\mathrm{a}}$ & $51.41 \pm 1.03^{\mathrm{a}}$ & $14.08 \pm 8.13^{\mathrm{b}}$ \\
Silvopastoril & $21.34 \pm 3.47^{\mathrm{a}}$ & $25.28 \pm 4.16^{\mathrm{a}}$ & $53.17 \pm 8.13^{\mathrm{a}}$ & $50.68 \pm 1.03^{\mathrm{a}}$ & $25.4 \pm 8.13^{\mathrm{a}}$ \\
Pastura & $17.42 \pm 3.47^{\mathrm{a}}$ & $23.84 \pm 4.16^{\mathrm{a}}$ & $49.89 \pm 8.13^{\mathrm{a}}$ & $50.29 \pm 1.03^{\mathrm{a}}$ & $26.47 \pm 8.13^{\mathrm{a}}$ \\
\hline
\end{tabular}

Error estándar= Hwcc:3.47, HӨcc:4.16, Zcc:8.13, Pt: 1.03, M:8.13. Medias con letra diferentes son significantes $(p>0,05)$. Prueba de Tukey.

La mayor porosidad total $(\mathrm{P}<0.05)$, fue para BS con respecto a los demás, demostrando éste sistema ser un gran retenedor de agua, puesto que la microporosidad sobrepasa a la macroporosidad (33.78 $>22.61 \%$ ), el anterior comportamiento obedece al su cobertura, formando una mayor capa de materia orgánica, lo cual ejerce efecto en la porosidad y almacenamiento de agua en suelo, (Tabla 5). En un estudio sobre el efecto protector del bosque, encontraron que, la 
materia orgánica es alta, así como hay bajas pérdidas de suelo y agua, en comparación los suelos labrados convencionalmente (Devine et al., 2014). Una alta diversidad de especies arbóreas en el BS, aporta gran cantidad de materia orgánica, mejorándose también la cantidad de macroporos promotores del drenaje y aireación.

Sin embargo, a pesar de que el sistema SSP no presentó diferencias con los otros sistemas en las variables que tienen que ver con la humedad, se obtuvo un valor de Pt ideal del $50.68 \%$, la cual está dividida en $25.28 \%$ de microporosidad, y $25.4 \%$ de macroporosidad, indicando que en este sistema los árboles ejercen influencia positiva en la pastura. Pignataro Netto et al., (2009) no observaron diferencias en los valores de PT a diferentes profundidades, siendo muy bajos $\mathrm{n}$ en pasturas de la región del Cerrado, coincidiendo con los de este estudio, ellos mencionan que posiblemente debido a la presencia de raíces vivas y descompuestas que dificultan la resistencia a la penetración. Por otra parte, en el MON, aunque presenta una Pt también cercana a la ideal, su macroporosidad es muy baja de $14,08 \%$, cercana a 10 , una $M<10 \%$, indica que puede existir restricciones para el desarrollo radicular de los cultivos, indicando algún grado de compactación (Tabla 5) (Batey y McKenzie, 2006). En el uso de MON, puede estar fallando la circulación de aire, se observa una alta susceptibilidad a la compactación en el corto plazo. Los sistemas intensivos están propensos a problemas de compactación, y baja infiltración de agua (Laureda et al., 2016). Los factores que afectan la resistencia del suelo han sido identificados por diferentes investigadores y se han establecido escalas para interpretar la su condición para el desarrollo de la rizosfera, según el RP de la capa edáfica: $\sin$ restricciones $(0 \leq R P \leq 0,9)$; leves restricciones $(0,9<R P \leq 1,4)$; moderadas a severas restricciones $(1,4<\mathrm{RP} \leq 2)$; restrictivo para enraizamiento $(\mathrm{RP}>2)$ (Zerpa et al., 2013).

Se observó que la influencia del uso del suelo en los sistemas evaluados, la resistencia a la penetración $(\mathrm{RP})$ a diferentes profundidades, puede afirmarse que ninguno presenta restricciones entre 20 a $80 \mathrm{~cm}$, sin embargo, existen ligeros incrementos en resistencia a la penetración entre los 40 a $80 \mathrm{~cm}$ para todos los 
sistemas, se compararon BS con MON, observándose comportamiento similar en estos dos sistemas y se pueden calificar "sin restricciones", por lo tanto se considera que estos valores limitan el desarrollo radicular (Figuras 1 y 2 ).
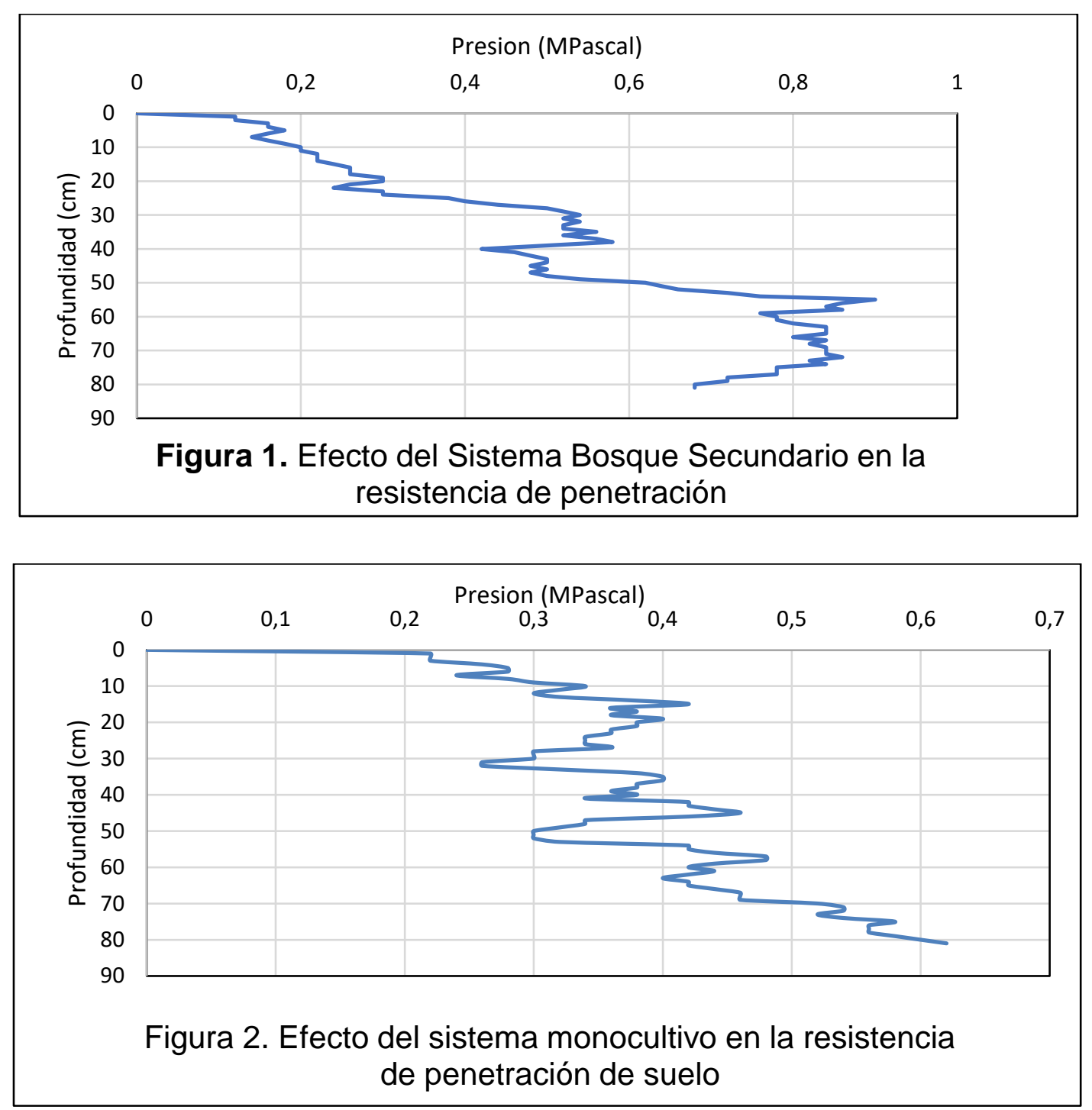

Los resultados difirieron también de lo encontrado por Ralisch et al., (2007), quienes estudiaron la RP en diferentes sistemas de manejo, determinando que este factor afecta las propiedades físicas de los suelos, influyendo en el crecimiento radical, la dinámica hídrica del suelo (infiltración, conductividad y almacenamiento), la captación de nutrientes y la emergencia de plántulas al momento de la siembra (Pariz et al., 2017). Se ha confirmado que la RP es más apropiada que la Da para la determinación de capas limitantes al crecimiento radical, porque presenta mayor 
sensibilidad en la detección de sectores diferenciados en grados de compactación, (Glab, 2011).

\section{CONCLUSIONES}

Las propiedades Da, RP y Macroporosidad, definieron que los sistemas de uso del suelo no presentan problemas de compactación, sin embargo, el sistema que mayor RP presentó fue PS con un promedio de $0.56 \mathrm{MPa}$ hasta los $80 \mathrm{~cm}$ de profundidad y el sistema con mayor Da y Macroporosidad fue MON con un valor de $1.32 \pm 0.30$ $\mathrm{g} / \mathrm{cm}^{3}$ y un contenido mayor $10 \%$ en los primeros $20 \mathrm{~cm}$ de profundidad respectivamente.

Los sistemas MON, PAS y SSP, presentaron texturas arenosas, indicando que son menos susceptibles a problemas de compactación. No obstante, el sistema de BS presenta textura franco limoso, considerándose un sistema frágil en perder sus condiciones ideales de suelo. El sistema BS presento las mejores condiciones físicas respecto a las variables DA y RP con valores de $1.15 \mathrm{gr} / \mathrm{cm}^{3}$ y 0,3 Mpa respectivamente.

\section{REFERENCIAS BIBLIOGRÁFICAS}

1. Alakukku, L.; Weisskopf, P.; Chamen, W.C.T.; Tijink, F.G.J.; Van Der Linden, J.P.; Pires, S.; Sommerf, C.; Spoor, G. 2003. Prevention strategies for field traffic induced subsoil compaction: A review Part 1. Machine/ soil interactions. Soil Till. Res., V: 73: 145-160.

Disponible:

http://www.scielo.br/scielo.php?script=sci nlinks\&ref=000093\&pid=S0100$6916200900030001000001 \& \operatorname{lng}=$ en

2. Andrade, P.; Upadhyaya S; Plouffe C; Poutre B. 2008. Development and field evaluation of a field-ready soil compaction profile sensor for real-time applications, Appl. Eng. Agric., 24 (6): 743-750.

3. Batey T, McKenzie D.C. 2006. Soil compaction: identification directly in the field. Soil Use and Management, 22 (2): 123-131. Disponible: https://www.researchgate.net/publication/227794719 Soil compaction Identificati on directly in the field

4. Bertol, I.; Schick, J.; Massariol, J, Reis, E.; Dily, I. 2000. Propriedades físicas de um cambissolo húmico álico afetadas pelo manejo do solo. Ciência Rural, Santa Maria, 30: 91-95.

5. Blainski, E, Fidalski J, Tormenta C y Muylaert R. 2008. Quantificação da degradação física do solo por meio da curva de resistência do solo à penetração. Revista Brasileira Ciência do Solo, 32: 975-983. Disponible: 
https://www.researchgate.net/publication/250033965 Quantificacao da degradac ao fisica do solo por meio da curva de resistencia do solo a penetracao

6. Cairo P, Reyes, A, Aro, R, Robledo, L. 2017. Efecto de las coberturas en algunas propiedades del suelo. Finca La Morrocoya, Barinas, Venezuela. Pastos y Forrajes, 40 (2): 127-134.

7. Camargo O., Alleoni, L. 1997. Compactação do solo e o desenvolvimento das plantas. Piracicaba: ESALQ, $132 \mathrm{p}$.

8. Devine, S.; Markewitz, D.; Hendrix, P.; Coleman, D. 2014.Soil aggregates and associated organic matter under conventional tillage, no-tillage, and forest succession after three decades. PLosOne. 9 (1):1-12.

9. Di Rienzo J.A., Casanoves F., Balzarini M.G., Gonzalez L., Tablada M., Robledo C.W. InfoStat versión 2014. Grupo InfoStat, FCA, Universidad Nacional de Córdoba, Argentina.

10. Fageria N, Baligar V. 2008. Chapter 7 Ameliorating soil acidity of tropical oxisols by liming for sustainable crop production. Advances in Agronomy, 99: 345-399.

11. Foloni, J., Calonego, J.; de Lima, S. 2003. Efecto de la compactación del suelo en desenvolvimiento aéreo y radical de cultivos de Brasilia. Revista Agropecuaria Brasileira, 38 (8): 947-953.

12. Food and Agriculture Organization (FAO). 2015. Carta mundial de los suelos. 12 p. Disponible: http://www.fao.org/soils- 2015/resources/fao-publications/es/

13. Food and Agriculture Organization (FAO). 2016. Estado Mundial del Recurso Suelo (Resumen Técnico). Roma. 92 p. Disponible: http://www.fao.org/3/a-i5126s.pdf

14. Glab, T. 2011. Effect of Soil Compaction on Root System Morphology and Productivity of Alfalfa (Medicago sativa L.). Polish Journal of Environmental Studies, 20 (6): 1473-1480.

15. González, L, Casanoves M, Di Rienzo F, Robledo J, Carlos W. Corporativo: Grupo InfoStat, InfoStat, versión 2008. Manual del usuario. Córdoba (Argentina). P.imprenta: Editorial Brujas. 334 p.

16. Hakansson, I.; Voorhees, W.R.; Riley, H. 1988 Vehicle and wheel factors influencing soil compaction and crop response in different traffic regimes. Soil and Tillage Research, Amsterdam, 11: 239-282.

17. Instituto Geográfico Agustín Codazzi (IGAC). 6aㅡ Ed. 2006. Métodos Analíticos del Laboratorio de Suelos. Bogotá D.C., Colombia: IGAC.

18. Instituto Geográfico Agustín Codazzi (IGAC). 2013. Guía de muestreo [en línea]. Bogotá D.C., Colombia: IGAC. Disponible en: https://www.igac.gov.co/sites/igac.gov.co/files/guiademuestreo.pdf

19. Instituto Geográfico Agustín Codazzi (IGAC). 2014. Manejo de suelos colombianos. Bogotá D.C., Colombia: IGAC.

20. Instituto Geográfico Agustín Codazzi (IGAC). 1a Ed. 2015. Suelos y tierras de Colombia. Subdirección de Agrología. Bogotá D.C., Colombia: IGAC.

21. Jaramillo D. 2002. Introducción a la Ciencia del suelo. Medellín: Universidad Nacional de Colombia. 619 p. Disponible en: http://www.bdigital.unal.edu.co/2242/1/70060838.2002.pdf

22. Laureda, D, Botta, G, Tolon A, Rosatto. 2016. Compactación del suelo inducida por la maquinaria en campos de polo en Argentina. Rev. Fac. Cienc. Agrar., Univ. Nac. Cuyo [online]. 48: 1: 79-99.

23. Lal, R. 2015. Restoring soil quality to mitigate soil degradation. Sustainability. 7: 5875-5895. Disponible en: https://www.mdpi.com/2071-1050/7/5/5875 
24. Lima, C., Silva, A.; Imhoff, S y Leão, T. 2004. Compressibilidade de um solo sob sistemas de pastejo rotacionado intensivo irrigado e não irrigado. Rev. Bras. Ciênc. Solo, Viçosa, $28 \quad$ (6): 945-951. Disponible en: https://www.rbcsjournal.org/article/soil-compressibility-under-non-irrigatedandhttp://dx.doi.org/10.1590/S0100-06832004000600002

25. London JR (Ed.) 2014. Booker Tropical Soil manual and handbook for soil survey an agricultural. Land evaluation in the tropics and subtropics. New York and London. 465 p. Disponible en: https://books.google.com.co/books?hl=en\&lr=\&id=xse2AgAAQBAJ\&oi=fnd\&pg=P P1\&dq=USDA.+(2014).+Soil+Survey+Manual.+Washington+DC:+USDA.\&ots=zU JCsYoJP4\&sig=E2vZsoa78n6TTrRbzaoM9RbnX5c\#v=onepage\&q=USDA.\%20(2 014).\%20Soil\%20Survey\%20Manual.\%20Washington\%20DC\%3A\%20USDA.\&f=f alse

26. Luciano, R, Adriano J, Da Costa A, Batistella B, Warmling M. 2012. Atributos físicos relacionados à compactação de solos sob vegetação nativa em região de altitude no Sul do Brasil. Rev. Bras. Ciênc. Solo, Viçosa, 36 (6): 1733-1744 Disponible en: http://www.scielo.br/pdf/rbcs/v36n6/07.pdf

27. Lüscher A, Mueller I, Soussana J, Rees R, Peyraud J. 2014. Potential of legumebased grassland-livestock systems in Europe: a review. Grass Forage Science, 69 (2): 206-228.

28. Mckenzie, R. H.; Research, S.; Agricultural Soil Compaction: Causes and Management, 2010. Alberta Agriculture and Rural. Consultado: 28/09/2018. Disponible en: http://www1.agric.gov.ab.ca/\$department/deptdocs.nsf/all/agdex13331/\$file/5101.pdf?OpenElement

29. Medina, C. 2016. Efectos de la compactación de suelos por el pisoteo de animales, en la productividad de los suelos. remediaciones. (Nota Corta). Rev Colombiana Cienc Anim, 8 (1):88-93.

30. Ministerio de Trabajo de Colombia (Mintrabajo). 2013. Perfil productivo Del municipio de Puerto López. $97 \quad$ p. $\quad$ Disponible: https://www.meta.gov.co/web/sites/default/files/ormet/2013/2.\%20PERFIL\%20PR ODUCTIVO\%20MUNICIPIO\%20DE\%20PUERTO\%20LOPEZ.pdf

31. Mouazen, A., Ramos H. 2009. Expanding implementation of an on-line measurement system of topsoil compaction in loamy sand, loam, silt loam and silt soils, Soil \&Tillage Research, 103: 98-104.

32. Moussadek, R., Mrabet, R., Dahan, A., Zouahri, M., Mourid, E., Van Ranst, E. 2014. Tillage system affects soil organic carbon storage and quality in Central Morocco. Applied and Environmental Soil Science. Article ID 654796 doi: $10.1155 / 2014 / 654796$.

33. Mur M, Balbuena R. 2014. Compactación de un suelo argiudol típico por tráfico en un sistema de producción de forrajes. Cienc. Suelo, Ciudad Autónoma de Buenos Aires, 32 (1): 1-12. Disponible en: https://dialnet.unirioja.es/ejemplar/374462

34. Noruega, M. A., Vélez, J. A. 2011. Evaluación de algunas propiedades físicas del suelo en diferentes usos. Nariño: Revista de ciencias Agrícolas.

35. Orellana G, Ortega S., Moreno, A. 2008. Fracción orgánica ligera del suelo como indicador agroecológico. Revista de Agricultura Orgánica, 2: 40-41.

36. Palosuo, T. 2008. Soil carbon modelling as a tool for carbon balance studies in forestry. Dissertationes Forestales 61 Department of Forest Ecology. Faculty of 
Agriculture and Forestry. University of Helsinki, 60 p. Disponible en: https://www.dissertationesforestales.fi/pdf/article1843.pdf

37. Pariz, C, Costa, C, Crusciol, A, Meirelles, P, Castilhos, A, Andreotti, M, Costa, N., Martello, J, Souza, D, Protes, V, Longhini, V y Franzluebbers, A. 2017. Production, nutrient cycling and soil compaction to grazing of grass companion cropping with corn and soybean. Nutr Cycl Agroecosyst, 108: 35-54.

38. Parra A, de Figueiredo E, de Bordonal, R. et al., 2019. Greenhouse gas emissions in conversion from extensive pasture to other agricultural systems in the Andean region of Colombia. Environment Development and Sustainability 21: 249.

39. Pignataro Netto, I; Kato, E; Goedert, W. 2009. Atributos físicos e químicos de um latossolo Vermelho-amarelo sob pastagens com diferentes históricos de uso. Revista Brasileira de Ciência do Solo, Viçosa, 3: 1441-1448.

40. Ralisch, R; Miranda, T; Okumura, R; Barbosa, G; Guimarães, M.; Scopel, E.; Balbino, L. 2007. Resistência a penetração de um latossolo vermelho amarelo do cerrado sob diferentes sistemas de manejo. Revista Brasileira de Engenharia Agrícola e Ambiental, 12 (4): 381-384.

41. Rodríguez, M. 2016. Stock de carbono del suelo, a escala local, en ocho sistemas de uso agrícola del piedemonte llanero, Universidad Nacional de Colombia Facultad de Ciencias Agropecuarias, Escuela de Posgrados Doctorado en Ciencias Agrarias, Palmira, $\quad$ Colombia, $140 \quad$ p. $\quad$ Disponible http://bdigital.unal.edu.co/51731/1/PhD.Maryory mayo.pdf

42. Silva, G, Lima H., Campanha, M., Gilkes, R., Oliveira, T. 2011. Soil physical quality of Luvisols under agroforestry, natural vegetation and conventional crop management systems in the Brazilian semi-arid region. Geoderma, 167-168: 61-70. Disponible en: https://ainfo.cnptia.embrapa.br/digital/bitstream/item/46818/1/APISoil-physical.pdf

43. Streck, C. A.; Reinert, D. J.; Reichert, J. M., Kaiser, D. R. 2004. Modificações em propriedades físicas com a compactação do solo causada pelo tráfego induzido de um trator em plantio direto. C. Rural, 34: 755-760.

44. Tavares Filho, J.; Tessier, D. 2009. Compressibility of oxisol aggregates under notill in response to soil water potential. Revista Brasileira de Ciência do Solo, 33: 1525-1533.

45. Valdés, A. 2010. Cómo controlan la erosión las raíces de las plantas. (Editorial), Revista de Divulgación Científica y Tecnológica de la Universidad Veracruzana, 23: 2. Disponible en: https://www.uv.mx/cienciahombre/revistae/vol23num2/articulos/erosion/.

46. Zerpa, G.; Sosa, O.; Berardi, J.; Bolatti, J. P.; Galindo, A., Maldonado, J. 2013.La resistencia mecánica a la penetración en pasturas. Agromensajes, 35: 64-68. 